\title{
CircRNAs open a new era in the study of cardiovascular disease (Review)
}

\author{
ZHIKANG YU ${ }^{1-3^{*}}$, QINGYAN HUANG ${ }^{1-3^{*}}$, QUNJI ZHANG ${ }^{1-3}$, HEMING WU $^{1-3}$ and ZHIXIONG ZHONG ${ }^{1-3}$ \\ ${ }^{1}$ Center for Precision Medicine, ${ }^{2}$ Guangdong Provincial Key Laboratory of Precision Medicine and Clinical Translational \\ Research of Hakka Population, ${ }^{3}$ Guangdong Provincial Engineering and Technology Research Center for Clinical \\ Molecular Diagnostics and Antibody Therapeutics, Meizhou People's Hospital (Huangtang Hospital), Meizhou Academy of \\ Medical Sciences, Meizhou Hospital Affiliated to Sun Yat-sen University, Meizhou, Guangdong 514031, P.R. China
}

Received May 28, 2020; Accepted October 26, 2020

DOI: $10.3892 /$ ijmm.2020.4792

\begin{abstract}
Circular RNAs (circRNAs) are non-coding RNAs that are found in the cytoplasm or stored in the exosomes, where they are not affected by RNA exonucleases. CircRNAs are widely expressed in mammalian tissues and cells. A number of studies have suggested that circRNAs are associated with the physiology and pathology of cardiovascular diseases (CVDs). Therefore, circRNAs have been considered as effector molecules and biomarkers in the cardiovascular system. The present review article summarizes the biological origin and roles of circRNAs as well as the available databases and research methods for their identification. Furthermore, it describes their regulatory mechanisms in cardiovascular physiology and pathology, including the regulation of atherosclerosis, immunity, cell proliferation, apoptosis and autophagy. In addition, the current review discusses the unresolved problems in circRNA research and the application of circRNAs in the treatment of CVDs. Finally, the CVD-associated circRNAs are also reviewed.
\end{abstract}

\section{Contents}

1. Introduction

2. Formation and classification of circRNAs

3. Biological functions of circRNAs

4. Databases and testing methods for circRNAs

Correspondence to: Dr Heming Wu or Professor Zhixiong Zhong, Center for Precision Medicine, Meizhou People's Hospital (Huangtang Hospital), Meizhou Academy of Medical Sciences, Meizhou Hospital Affiliated to Sun Yat-sen University, 63 Huangtang Road, Meijiang, Meizhou, Guangdong 514031, P.R. China

E-mail: wuheming1986@126.com

E-mail: zhongzhixiong01@126.com

*Contributed equally

Key words: circular RNA, heart and vascular cells, cardiovascular disease, regulatory mechanism, biomarkers
5. Expression of circRNAs in the heart

6. Expression of circRNAs in cells of the cardiovascular system

7. Regulatory mechanisms of circRNAs on CVD

8. Perspectives and conclusions

\section{Introduction}

It is estimated that only $20 \%$ of the nucleic acids in the human genome are encoded into proteins, while the rest, the so called non-coding nucleic acids, are considered as the 'dark matter' of the genome (1). However, several studies have shown that these non-coding RNAs are involved in important biological processes, such as cell proliferation, differentiation, apoptosis, metabolism, senescence, and especially in post-transcriptional regulation (1-3). Circular RNAs (circRNAs) were first discovered in the early 1970 s $(4,5)$, however, they have been poorly studied for nearly three decades due to the limited available technology. Recent advances in RNA sequencing technologies have promoted the identification of an increasing number of circRNAs $(3,6-8)$. Several studies have shown that circRNAs can compete with mRNAs for the target binding sites on microRNAs (miRNAs) to affect gene expression. However, the function of the majority of circRNAs remains unclear. In 2013, Hansen et al (9) reported the overlapping co-expression of circRNA ciRS-7 and miR-7 in murine brain tissues. In addition, it was also demonstrated that the circRNA sex-determining region Y-9 (SRY-9) could act as a sponge for miR-138, thus providing the first solid evidence for the biological roles of circRNAs. To date, $>183,000$ circRNAs have been identified from human transcripts, to the best of our knowledge (10). The role of circRNAs in different tissues and different diseases have also been gradually revealed (11). The cardiovascular system is considered as one of the two major vascular systems in the human body. Cardiovascular diseases (CVDs) remain the leading cause of death worldwide. A study found that the circRNAs solute carrier family 8 member A1 (SLC8A1), ataxin 10 (ATXN10), SWI/SNF-related matrix-associated actin-dependent regulator of chromatin subfamily A member 5 (SMARCA5), chromodomain Y (CDY) and myoblast determination protein 1 (MyoD) are 
associated with myocardial differentiation (12). In addition, several circRNAs are involved in the pathological process of atherosclerosis (AS) (13). Furthermore, stress stimuli such as hypoxia and high temperature have been associated with the expression of cardiovascular-related factors (14-16). Emerging evidence has shown that circRNAs are involved in the progression of CVD, and are therefore considered promising targets for diagnosis and treatment. The present review article aimed to summarize the current knowledge on the biological aspects of circRNAs and highlight the current research progress on the mechanisms underlying the function of circRNAs in the physiology and pathology of the cardiovascular system.

\section{Formation and classification of circRNAs}

It is considered that circRNAs are derived from the variable splicing of precursor mRNAs (pre-mRNA), mediated by RNA polymerase II (17-19). However, to the best of our knowledge, the mechanism underlying the formation of circRNAs remains to be elucidated.

CircRNAs can be cyclized by RNA binding protein (RBP) (20). For example, the RNA binding proteins, mannose-binding lectin (17), RBP quaking (21) and fused in sarcoma (22) are known to drive the folding and formation of precursor mRNAs of circRNAs. The aforementioned RBPs bind to introns near the splicing site to promote the formation of dimers upstream and downstream of the cyclic exons to aid their splicing, thus leading to the production of circRNAs.

CircRNAs may also be formed by complementary pairing of flanking introns located in dorsal exons, such as short interspersed nuclear elements or non-repetitive complementary sequences $(23,24)$. Among them, Alu repetitive elements are widespread in the expressed RNA and in the non-coding parts $(25,26)$. Alu repeats serve a key role in the formation of circRNAs, with $>1$ million copies in the genome (24). It is considered that the probability and intensity of the reverse intron folding, mediated by the repeats encompassed in the pre-mRNA, may also determine the frequency of reverse splicing $(25,27)$.

Lariat-driven circularization is another important process of circRNA formation (28). Exon skipping occurs when the pre-mRNA is spliced, resulting in a lariat intermediate containing exons and introns. Subsequently, the internal splicing of the lariat leads to the removal or retention of introns, thus resulting in the formation of exon or exon-intron circRNAs (29-32).

Based on their biogenesis, circRNAs are divided into exon circRNAs, encompassing a single exon or multiple exons, exon-intron circRNAs, and intron circRNAs (Fig. 1). The majority of circRNAs belong to the first subtype and mainly exist in the cytoplasm (33). However, a few circRNAs are produced in the nucleus and can be secreted into the body fluids, such as the serum, saliva and cerebrospinal fluid (34-36). The exon-intron circRNAs are mainly located in the nucleus and interact with RNA polymerase II to promote the transcription of their parental genes (37). Finally, the intron circRNAs exist in the nucleus (38).

\section{Biological functions of circRNAs}

Gradually, circRNAs have been found to exert more specific biological functions, and their mechanism of action remains in the focus of attention $(39,40)$. To date, several functions of circRNAs have been verified (Fig. 1).

MicroRNA sponges. Apart from a small number of circRNAs generated by intron circularization, which are located in the nucleus, the majority are mainly located in the cytoplasm (41). Several studies have shown that circRNAs can compete with mRNAs for binding to miRNAs located in the cytoplasm, thereby regulating the expression of mRNAs $(3,9)$. ciRS-7/cerebellar degeneration-related protein 1 antisense RNA (CDRlas) sponging miR-7 and mouse SRY sponging miR-138 are considered as the most representative circRNAs supporting this biological function (3). Notably, only a few circRNAs have been identified to encompass multiple target binding sites for miRNAs (42). Furthermore, the binding of circRNAs by miRNAs may initiate circRNA decay (43). It has been also demonstrated that the CDR1as-miR-7 axis is modulated by Cyrano, a long non-coding RNA (lncRNA) (44). In the non-coding regulatory network, miR-671 shares a highly complementary sequence with CDRlas, which triggers the rare argonaute2-mediated RNA cleavage targeted by miRNAs in mammals and even vertebrates (43). The aforementioned findings indicate that circRNA-miRNA interactions not only mediate miRNA sequestration, but also exert other functions that are worth studying.

Competition with pre-mRNAs for cleavage and splicing during transcription. CircRNAs are formed as a result of the pre-mRNA atypical splicing, whereas mRNA is the final product of the pre-mRNA typical linear splicing (45). A study revealed that improving the efficiency of typical linear splicing could lead to a significant reduction in the number of generated circRNAs (17). When the length of the intron flanking the exon is longer, the efficiency of typical linear splicing is significantly decreased, and cyclization occurs (46). These findings indicate that circRNAs can compete with pre-mRNA during transcription.

CircRNAs regulate transcription and chromatin interactions, and act as protein sponges. CircRNAs located in the nucleus can bind RNA polymerase II or U1 small nuclear ribonucleoprotein to regulate the transcription of their parental genes $(47,48)$. A study has shown that the expression of the parental genes is significantly attenuated when circRNAs are knocked out (47). Furthermore, back-splicing was observed in RNAs transcribed from centromeric retrotransposons in maize, while the resulting circular CRM1 RNAs could bind to maize centromeres through R-loops to promote the formation of chromatin loops. Previous studies have demonstrated that QKI attenuated doxorubicin (DOX)-induced cardiotoxicity via binding to titin ( $\mathrm{Ttn}$ )-, formin homology 2 domain containing 3 (Fhod3)- and striatin 3 (Strn3)-derived circRNAs expressed in the heart (49). In addition, circRNAs contain binding sites for the RBPs of the host and can regulate their expression. For example, circRNA zinc finger protein 609 (ZNF609) can regulate the proportion of phosphorylated $(\mathrm{p})-\mathrm{Rb} / \mathrm{Rb}$ and the levels of p-protein kinase B (Akt), thus affecting the G1/S phase progression in cells (50).

Translation of circRNAs. CircRNAs were originally defined as non-coding RNAs (51). However, it has been reported 


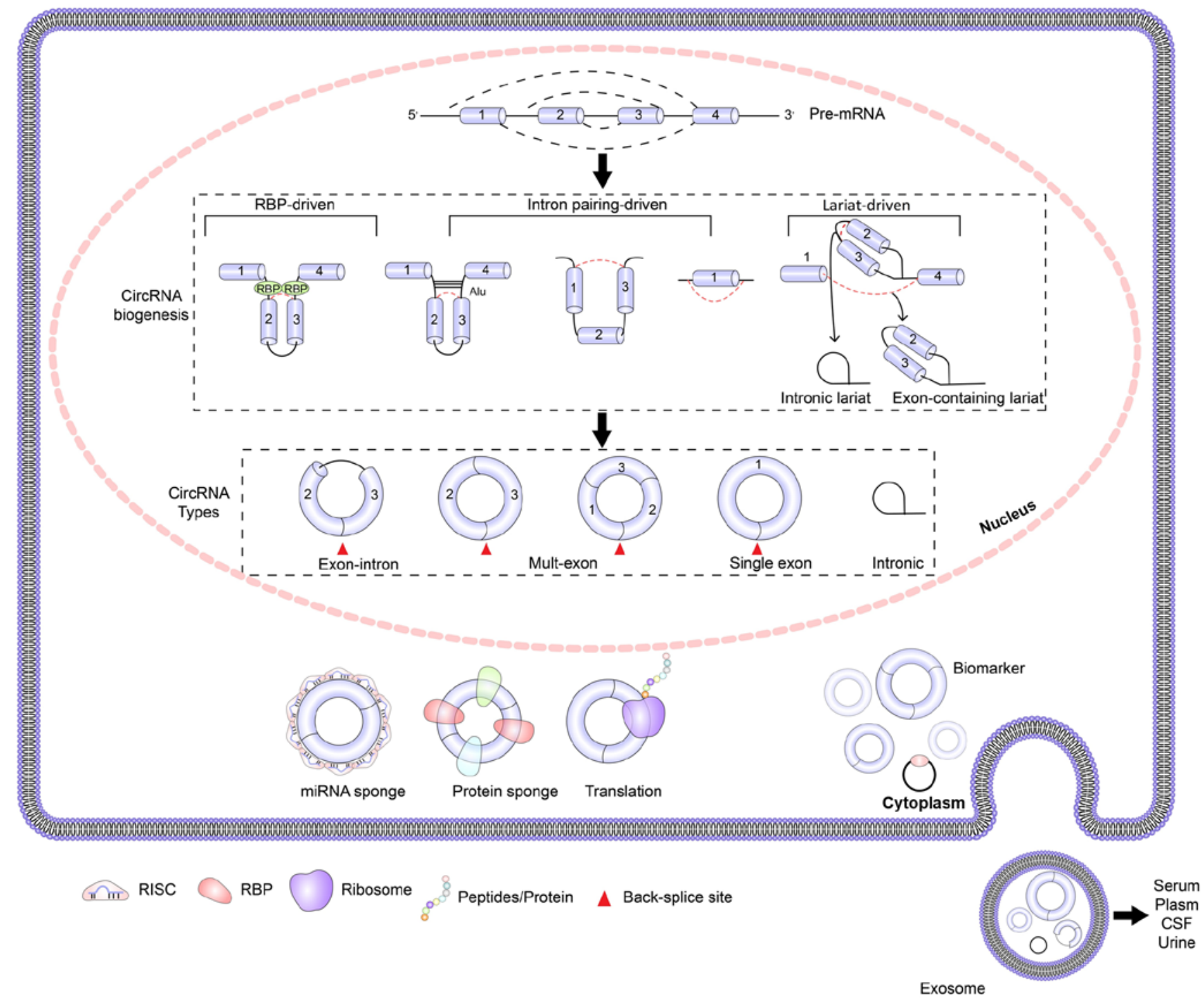

Figure 1. Schematic representation showing the biogenesis and function of circRNAs. CircRNA formation models: i) Intron pairing-driven circularization; ii) RBP-driven circularization; and iii) lariat-driven circularization. Types of circRNA: i) Single exon, intronic; ii) exon-intron; and iii) multi-exon. CircRNA functions: i) miRNA sponge; ii) scaffold for proteins, iii) translation; and iv) biomarker. CircRNA, circular RNA; RBP, RNA binding protein; miRNA, microRNA; RISC, RNA-induced splicing complex; CSF, cerebrospinal fluid.

that translation may be carried out by the internal ribosome entry site (IRES) of circRNAs. For example, the untranslated region of endogenous circRNA ZNF609 can act as an IRES in a splicing-dependent manner, suggesting that circRNA ZNF609 can drive IRES-dependent translation (52). A study revealed that consensus N6-methyladenosine (m6A) motifs are enriched in circRNAs, while a single m6A site is sufficient to initiate translation. This m6A-dependent translation requires eukaryotic translation initiation factor $4 \mathrm{~F}$ and the m6A reader, YTH N6-methyladenosine RNA binding protein 3 (53). Additionally, m6A-dependent translation can be enhanced by methyltransferase like $3 / 14$, inhibited by demethylase obesity-associated protein and is upregulated in response to heat shock (53). m6A-driven circRNA translation commonly occurs, and hundreds of endogenous circRNAs exert translational capability (53). The translation capacity of circRNAs is not considered as their common function, however, further study on this field may provide revolutionary changes.

\section{Databases and testing methods for circRNAs}

Several laboratories and institutions have developed specialized databases and analytical tools to study circRNAs $(10,54-65)$ (Table I). These databases provide the convenience to further investigate the already identified circRNAs. RNA sequencing analysis of rRNA-depleted total RNAs and microarrays are currently applied for the discovery of novel circRNAs (66). Nevertheless, the verification of the predicted circRNAs by in vitro/in vivo experiments is always necessary. Several widely used molecular biology techniques have been applied to detect and verify circRNAs, including northern blot analysis, which is considered as the gold standard for circRNAs verification $(3,30)$, reverse transcription (RT)-PCR, which is the most commonly used and basic verification technique (17), quantitative PCR which is used to identify and quantitatively measure differentially expressed circRNAs, droplet digital PCR, which is performed to quantify the expression of circRNAs in challenging samples such as plasma $(67,68)$, and fluorescence in situ 
Table I. List of the main circRNA databases.

\begin{tabular}{|c|c|c|c|c|c|}
\hline First author, year & Database & Function & URL & Last update & (Refs.) \\
\hline Glažar et al, 2014 & CircBase & $\begin{array}{l}\text { Facilitates the identification } \\
\text { of circRNA in sequencing } \\
\text { data }\end{array}$ & http://www.circbase.org & 2017 & $(54)$ \\
\hline Panda et al, 2018 & CircInteractome & $\begin{array}{l}\text { Predicts the interaction } \\
\text { between circRNA and RNA } \\
\text { binding proteins and miRNA }\end{array}$ & http://circinteractome.nia.nih.gov/ & 2018 & $(55)$ \\
\hline Liu et al, 2016 & CircNet & $\begin{array}{l}\text { Provides expression data of } \\
\text { circRNA and regulatory } \\
\text { network of circRNA-miRNA } \\
\text { in different tissues }\end{array}$ & http://circnet.mbc.nctu.edu.tw & 2016 & $(56)$ \\
\hline Zheng et al, 2016 & Deep Base & $\begin{array}{l}\text { Provides small RNAs, } \\
\text { long non-coding RNAs and } \\
\text { circRNAs for deep } \\
\text { sequencing of different } \\
\text { species and tissues }\end{array}$ & http://rna.sysu.edu.cn/deepBase & 2016 & $(57)$ \\
\hline Liu et al, 2019 & Circbank & $\begin{array}{l}\text { Comprehensive database } \\
\text { with circRNAs standard } \\
\text { naming }\end{array}$ & http://http://www.circbank.cn & 2019 & $(58)$ \\
\hline Xia et al, 2018 & CSCD & $\begin{array}{l}\text { Database of specific } \\
\text { circRNAs associated with } \\
\text { cancer }\end{array}$ & http://gb.whu.edu.cn/CSCD & 2016 & $(59)$ \\
\hline Ghosal et al, 2013 & Circ2Traits & $\begin{array}{l}\text { CircRNAs database related } \\
\text { to human diseases }\end{array}$ & http://gyanxet-beta.com/circdb & 2013 & $(60)$ \\
\hline Li et al, 2014 & StarBase & $\begin{array}{l}\text { Large-scale CLIP-Seq } \\
\text { decoding the Interaction } \\
\text { Networks }\end{array}$ & $\begin{array}{l}\text { http://starbase.sysu.edu.cn/starbase } \\
\text { 2/index.php }\end{array}$ & 2019 & $(61)$ \\
\hline Dong et al, 2018 & CIRCpedia & $\begin{array}{l}\text { Allows comparison of } \\
\text { circRNA expression in } \\
\text { tissues and cell lines and } \\
\text { classification of alternative } \\
\text { reverse splicing and } \\
\text { alternative splicing }\end{array}$ & $\begin{array}{l}\text { http://www.picb.ac.cn/rnomics/ } \\
\text { circpedia/ }\end{array}$ & 2018 & $(10)$ \\
\hline Wang, 2018 & HppRNA & $\begin{array}{l}\text { Meets the needs of large } \\
\text { sample RNA-Seq system } \\
\text { analysis for circRNAs } \\
\text { research }\end{array}$ & https://sourceforge.net/projects/hpprna/ & 2018 & $(62)$ \\
\hline Wu et al, 2020 & CircAtlas 2.0 & $\begin{array}{l}\text { Provides functional } \\
\text { annotation and prioritization } \\
\text { of circRNAs for further } \\
\text { verification and functional } \\
\text { research }\end{array}$ & http://circatlas.biols.ac.cn/ & 2020 & $(63)$ \\
\hline Cai et al, 2020 & VirusCircBase & $\begin{array}{l}\text { The first comprehensive } \\
\text { database of circular RNA } \\
\text { molecules in viruses }\end{array}$ & $\begin{array}{l}\text { http://www.computationalbiology.cn/ } \\
\text { ViruscircBase/home.html }\end{array}$ & 2019 & $(64)$ \\
\hline Chen et al, 2016 & CircRNADb & $\begin{array}{l}\text { Provides comments on } \\
\text { chromosome location, } \\
\text { source gene, genome length, } \\
\text { protein coding }\end{array}$ & http://reprod.njmu.edu.cn/CircRNAdb & 2016 & $(65)$ \\
\hline
\end{tabular}

circRNA, circular RNA. 
hybridization, which is used to detect circRNA localization and interactions. Furthermore, overexpression and silencing techniques are performed to investigate the role of circRNAs in vitro or in vivo. For example, for the overexpression experiments, following amplification of a target DNA sequence encompassing the characteristic flanking Alu sequences, the sequence is digested with a restriction endonuclease and ligated to the pEGFP-C1 vector. Subsequently, the overexpression plasmid is transfected into the corresponding cell sample, thus resulting in the overexpression of the circRNAs $(69,70)$. Additionally, for silencing experiments, small interfering RNA (siRNA) sequences complementary to the back-splice junction are synthesized and the RNA-induced silencing complex specifically targets circRNA, leading to its cleavage and degradation, thus silencing its expression (52,71). Notably, the effectiveness of siRNAs depends on transfection efficiency, and these RNAs only temporarily inhibit the expression of their corresponding targets (72). However, short hairpin RNAs (shRNAs) or Ago shRNAs exert fewer off-target effects, thus resulting in more stable target-circRNA knockdown (73). The progress on studies concerning the effects of circRNAs will promote the merging of specific versions of databases to expand research in the field of circRNAs.

\section{Expression of circRNAs in the heart}

Several studies have reported the comprehensive expression profiles of circRNAs in rat, murine and human hearts (74-76), including the first study on the development and expression of circRNAs in human induced pluripotent stem cell-derived cardiomyocytes (76). A study reported for the first time a catalog of 575 candidate circRNAs in murine hearts. Among them, several candidate genes were found to be associated with different diseases (74). Furthermore, another study extensively compared human, rat and murine circRNA conservation in a specific tissue (75). Only $10 \%$ of these circRNAs were conserved among the three species. In addition, this study demonstrated that the overall circRNA expression was significantly decreased in the heart of adult animals compared with neonatal rats. The expression of circRNAs in the human heart was approximately twice the baseline level of adult rats and mice. A study analyzed the characteristics of circRNA expression in 12 human hearts, 25 mouse hearts and across a 28-day differentiation time-course from human embryonic stem cell-derived cardiomyocytes (76). A total of 15,318 and 3,017 circRNAs were identified in human and murine myocardial tissue, respectively. The expression abundance of these circRNAs was basically associated with their cognate linear RNA. The parental genes of circRNAs with the highest content were also considered as key cardiac genes, such as ryanodine receptor 2 (Ryr2), Ttn and dystrophin. Among them, circRNA SLC8A1-1 was the most abundantly expressed circRNA in the myocardium, whereas 402 different circRNA isoforms were expressed from the Ttn gene locus. These circRNAs were dynamically and highly expressed during heart development. In addition, it has been reported that the circRNAs SLC8A1, circ-calcium voltage-gated channel subunit $\alpha 1 \mathrm{D}$, SPHKAP and $\alpha$ kinase 2 are also specifically expressed in the heart (77). Another study showed that the circRNAs ATXN10, SMARCA5, CDY, MYOD, SLC8A1, ATXN7 and PHF2 were involved in cardiomyocyte differentiation (12). Furthermore, a notable study investigated the changes in the expression of lncRNAs, circRNAs and protein-coding genes via recording the RNA sequencing data at sequential stages of cardiomyocyte differentiation (78). The stages of differentiation studied were the following: Undifferentiated cardiomyocytes, mesoderm cardiomyocytes, cardiac progenitors and differentiated cardiomyocytes. This study described the dynamic expression pattern of non-coding RNAs during cardiogenesis, where the expression of circRNA SLC8A1 was gradually upregulated during cardiac differentiation. CircRNAs are derived from exons containing annotated start codons, which are also termed AUG circRNAs (79). Jakobi et al (80) described the AUG circRNAs in a heart model system for the first time, to the best of our knowledge. The authors demonstrated that m6A methylation was enriched in AUG circRNAs. They also reported the potential negative regulatory effect of AUG circRNAs on the translation of host genes. A recent study demonstrated that 40 ribosome-associated cardiac circRNAs could be translated into microproteins with different biological functions (81). Among them, the circRNAs CASP8 and FADD like apoptosis regulator, SLC8A1, myosin binding protein C3 and Ryr2 were reported for the first time. Therefore, the research on circRNAs will open a new chapter in heart biology.

\section{Expression of circRNAs in cells of the cardiovascular system}

It is understood that cardiomyocytes play an important role in the cardiovascular system $(75,82)$. It has been reported that several circRNAs, such as hsa_circ_0001879, hsa_circ_0004104 and hsa_circTCF25, can be isolated from peripheral blood mononuclear cells, serving as biomarkers of coronary artery disease (CAD) $(83,84)$. Another study revealed that the expression of circANRIL subtypes were inversely correlated with the risk and the severity of AS (85). Furthermore, a recent study documented for the first time the association between the expression of circRNAs and atrial fibrillation (86). Therefore, a total of five circRNAs were identified with notable biological significance, including chr9:15474007-15490122, chr16:75445723-75448593, hsa_circ_0007256, chr12:56563313-56563992 and hsa_ circ_0003533. Regarding vascular endothelial cells, circRNAs may directly or indirectly affect the occurrence of CVDs through functional changes, such as endothelial cell apoptosis, endothelial-mesenchymal transition and angiogenesis (87-90). For example, in vitro results demonstrated that the expression of circRNA ZNF292 in human umbilical vein endothelial cells (HUVECs) was significantly upregulated, suggesting that and circRNA ZNF292 could exert pro-angiogenic effects (71). Li et al (91) reported that the expression of hsa_circ_0003575 and hsa_circ_0003204 were significantly increased in oxidized low-density lipoprotein (ox-LDL)-induced HUVECs. Furthermore, hsa_circ_0003575 silencing significantly inhibited apoptosis and promoted proliferation and angiogenesis of ox-LDL-induced HUVECs. Yang et al (92) found that the circRNA HECT, C2 and WW domain containing E3 ubiquitin protein ligase 2 induced the expression of autophagy-related 5 (ATG5) in methamphetamine- or lipopolysaccharide (LPS)-induced human brain microvascular endothelial cells 
via binding to miR-30d, and ATG5 was shown to affect the endothelial-mesenchymal transition in an autophagy-independent manner. Liu et al (93) revealed the mechanism underlying the microvascular dysfunction mediated by diabetes. This mechanism also mediates the biological changes in human retinal vascular endothelial cells following circRNA PWWP domain-containing protein $2 \mathrm{~A}$ transfer from vascular pericytes to HUVECs through exosomes (93). Vascular smooth muscle cells (VSMCs) are another notable type of cardiovascular cells. A recent study reported that the circRNA low-density lipoprotein receptor-related protein 6 (Lrp6) can promote the phenotypic transformation of VSMCs via inhibiting miR-145, while silencing of circRNA Lrp6, using shRNA technology, attenuated intimal hyperplasia in a mouse carotid artery injury model (94). Additionally, Zheng et al (95) demonstrated that hsa_circ_000595 is upregulated in human aortic aneurysm patients and hypoxic VSMCs. In addition, hsa_circ_000595 can directly target cyclooxygenase 2 , hypoxia-inducible factor $1 \alpha(\mathrm{HIF}-1 \alpha)$ and nuclear factor- $\kappa \mathrm{B}$ to promote its biological function in regulating cell apoptosis via targeting miR-19a. By contrast, hsa-circ-000595 knockdown significantly attenuated apoptosis (95). Sun et al (96) reported that angiotensin II inhibits apoptosis of mouse aortic smooth muscle cells through regulating the circNRG-1/miR-193b-5p/NRG-1 axis. Several circRNAs have been identified to regulate the phenotypic transformation of VSMCs, including WD repeat domain 77, diaphanous related formin 3 and actin $\alpha 2$ (97,98). Regarding cardiomyocytes and cardiac fibroblasts, Gupta et al (49) demonstrated that QKI could inhibit DOX-induced cardiotoxicity via regulating the expression of the circRNAs Ttn, Fhod3 and Strn3. Furthermore, QKI could regulate circRNA expression to protect cardiomyocytes against adriamycin-induced injury in primary rat, $\mathrm{H} 9 \mathrm{C} 2$ and $\mathrm{HL}-1$ cardiomyocytes in vitro. CircRNAs are also considered as potential targets for treating cardiac fibrosis (99-101). Therefore, a study showed that the expression of circRNA_000203 of the myosin I9A transcripts was increased in the myocardium of diabetic mice and in angiotensin II-induced cardiac fibroblasts (102). It has been also revealed that the circRNA_000203/miR-26b-5p/ collagen type I $\alpha 2$ and connective tissue growth factor axes regulate the anti-fibrotic effects of cardiac fibroblasts (102). Wang et al (82) demonstrated that the mitochondrial fission and apoptosis-related circRNA (MFACR) could mediate cardiomyocyte mitochondrial fission and apoptosis via regulating the MFACR/miR-652-3p/mitochondrial trifunctional protein 18 axis. These findings provided novel knowledge for understanding the molecular events associated with mitochondrial fission. The circRNAs involved in the cardiovascular system are listed in Table II.

\section{Regulatory mechanisms of circRNAs on CVD}

It is understood that circRNAs are involved in numerous pathological mechanisms underlying CVDs, such as AS, immune response, apoptosis, cell proliferation, autophagy, cell senescence and myocardial repair (103-109). Emerging evidence has suggested that circRNAs play an important role in the occurrence and development of CVD (110). The documented regulatory mechanisms of circRNAs on CVDs are listed in Fig. 2.
Role of circRNAs in cell proliferation. The proliferation of cells involved in the cardiovascular system plays an important role in the development of CVDs, such as hypertension, AS and restenosis (110). CircRNAs serve an important role in the development of CVDs $(103,111)$. Shen et al (103) demonstrated that circRNA-0044073 is highly expressed in patients with AS. In addition, it was shown that circRNA-0044073 promotes the proliferation and invasion of human umbilical vein smooth muscle cells and HUVECs, and accelerates the development of AS via targeting miR-107 and activating the Janus kinase/signal transducer and activator of transcription. Wang et al (112) found that the circRNA homeodomain interacting protein kinase 3 could act as a sponge to inhibit miR-29a activity, resulting in increased insulin-like growth factor 1 expression, which in turn could attenuate the dysfunction of oxidative stress-induced cerebral microvascular endothelial cells (CMVECs). Additionally, the circRNA HIPK3 promoted the proliferation and survival of CMVECs under hypoxic conditions. Another study demonstrated that HIPK3 could sponge miR-29b-3p to regulate the proliferation, migration and development of cardiac fibroblasts in angiotensin II-induced myocardial fibrosis (111).

Role of circRNAs in cardiomyocyte apoptosis. Cardiomyocyte apoptosis is an important factor affecting the occurrence and development of CVDs (104,113-115). Geng et al (104) investigated the effect of the co-expression of circ-Cdrlas with miR-7a in mouse myocardial infarction injury model. Cdrlas overexpression upregulated the expression of poly (ADP-ribose) polymerase and specific protein 1 to promote cardiomyocyte apoptosis, but was then reversed by miR-7a overexpression. Li et al (113) suggested that reactive oxygen species could upregulate circ-NCX1, which in turn could promote cardiomyocyte apoptosis. Mechanistically, circ-NCX1 could competitively bind to $\mathrm{miR}-133 \mathrm{a}-3 \mathrm{p}$ to increase the expression levels of the pro-apoptotic protein gene cell death-inducing protein 1 (CDIP1). Therefore, circNCX1 knockdown reduced the expression of CDIP1 in murine cardiomyocytes and heart tissues, thereby improving the condition of myocardial ischemia-reperfusion injury. Several other circRNAs have been identified to play a role in promoting cardiomyocyte apoptosis, including circ-MFACR and circRNA_0010729 $(82,114)$.

Role of circRNAs in the regulation of AS. As early as 2010, a study revealed that the INK4/ADP ribosylation factor (ARF) gene locus is adjacent to the chromosome 9p21, which is associated with CAD (116). Circ-ANRIL, the antisense transcript of the INK4/ARF gene, was shown to be regulated by a single nucleotide polymorphism in the 9p21 chromosome and is associated with the risk of AS. Song et al (117) demonstrated that circ-ANRIL downregulation could prevent coronary AS via attenuating vascular endothelial cell apoptosis and the expression of inflammatory factors. However, another study suggested that circ-ANRIL exerts a protective effect against AS (85). The proportion of circ-ANRIL/lncRNA ANRIL in patients with coronary heart disease is higher, and patients with increased expression of circ-ANRIL rarely develop coronary heart disease. Furthermore, circ-ANRIL could induce nucleolar stress and p53 activation in VSMCs and macrophages, thus inducing and inhibiting apoptosis and proliferation, 


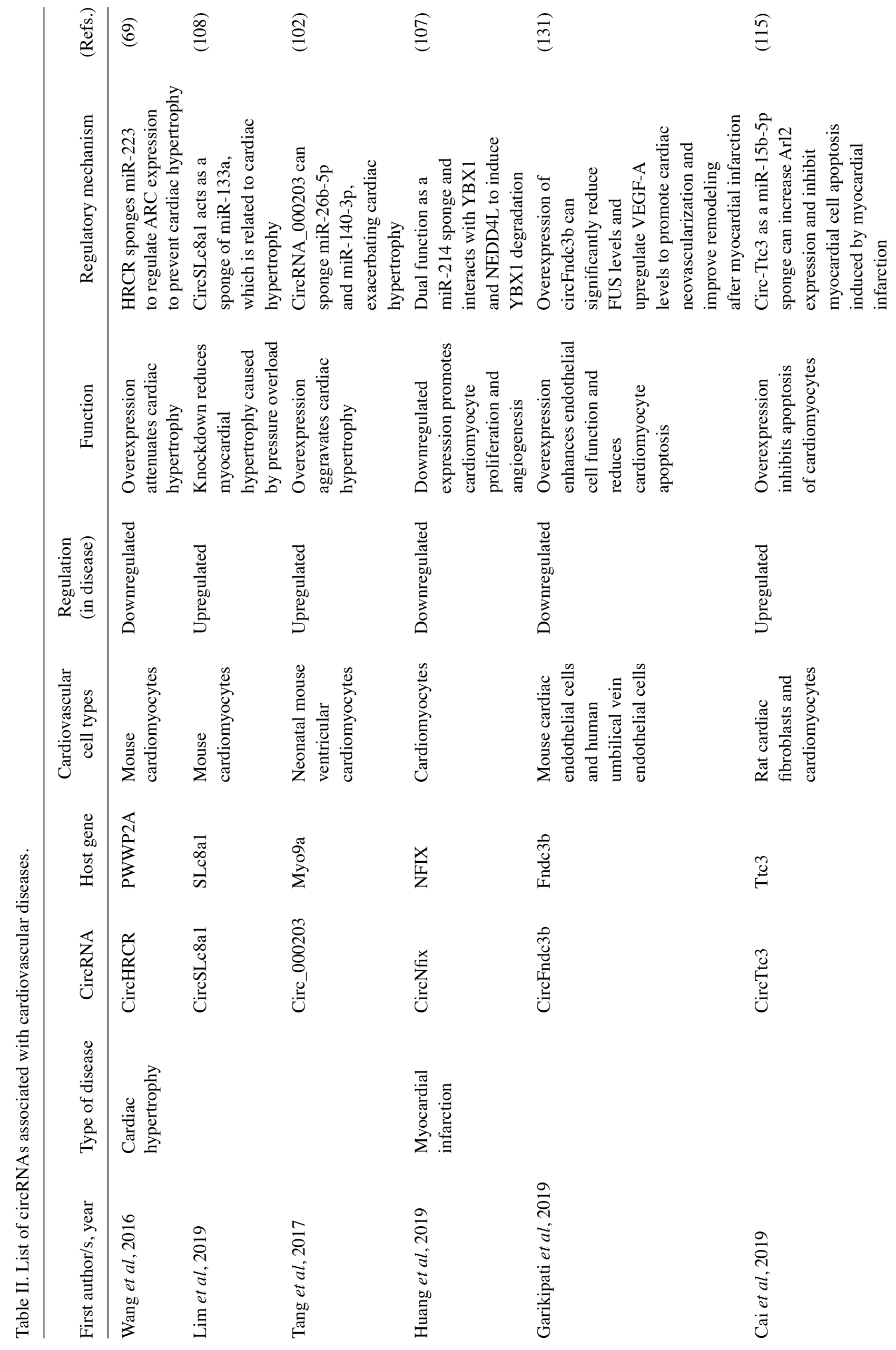




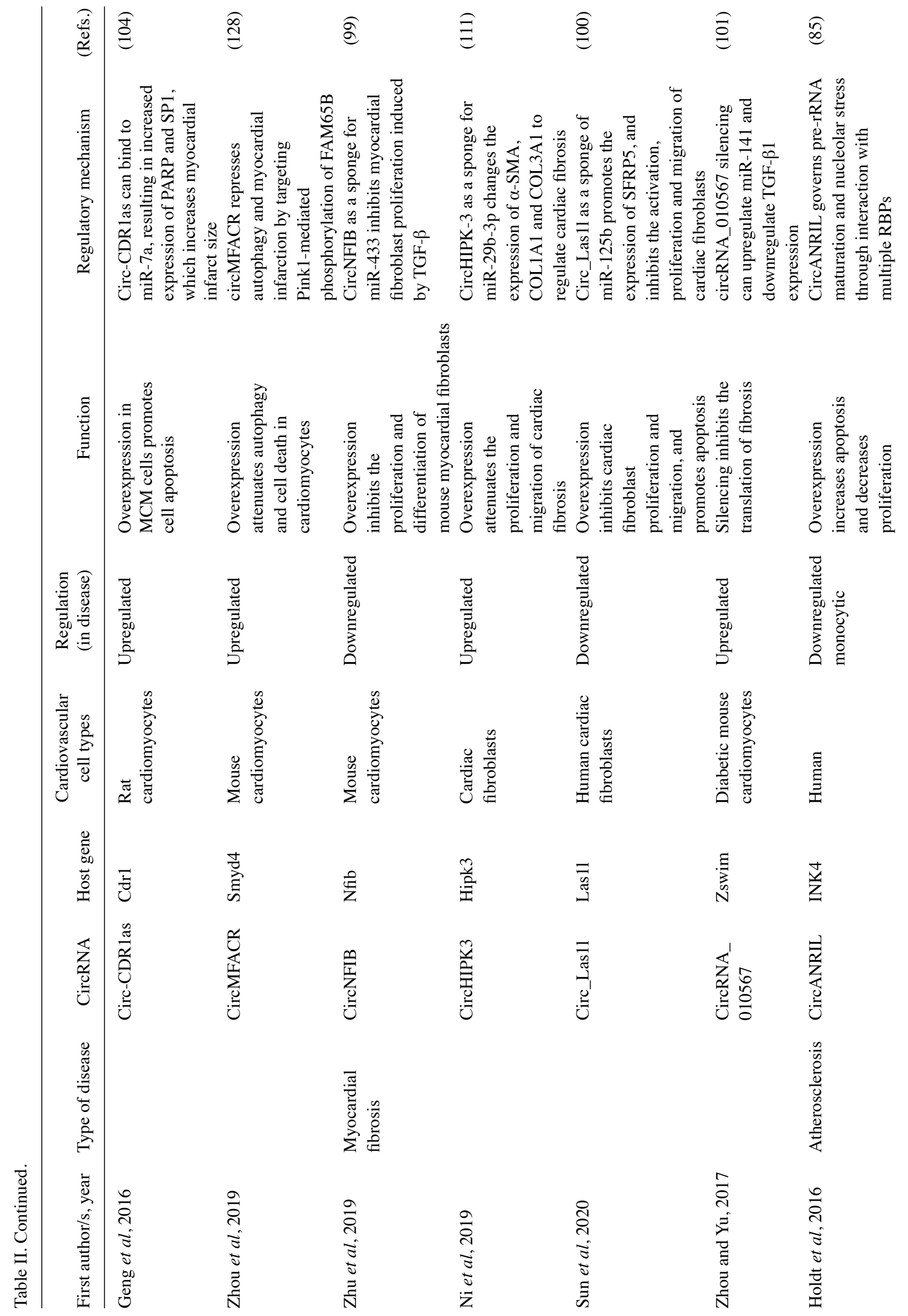




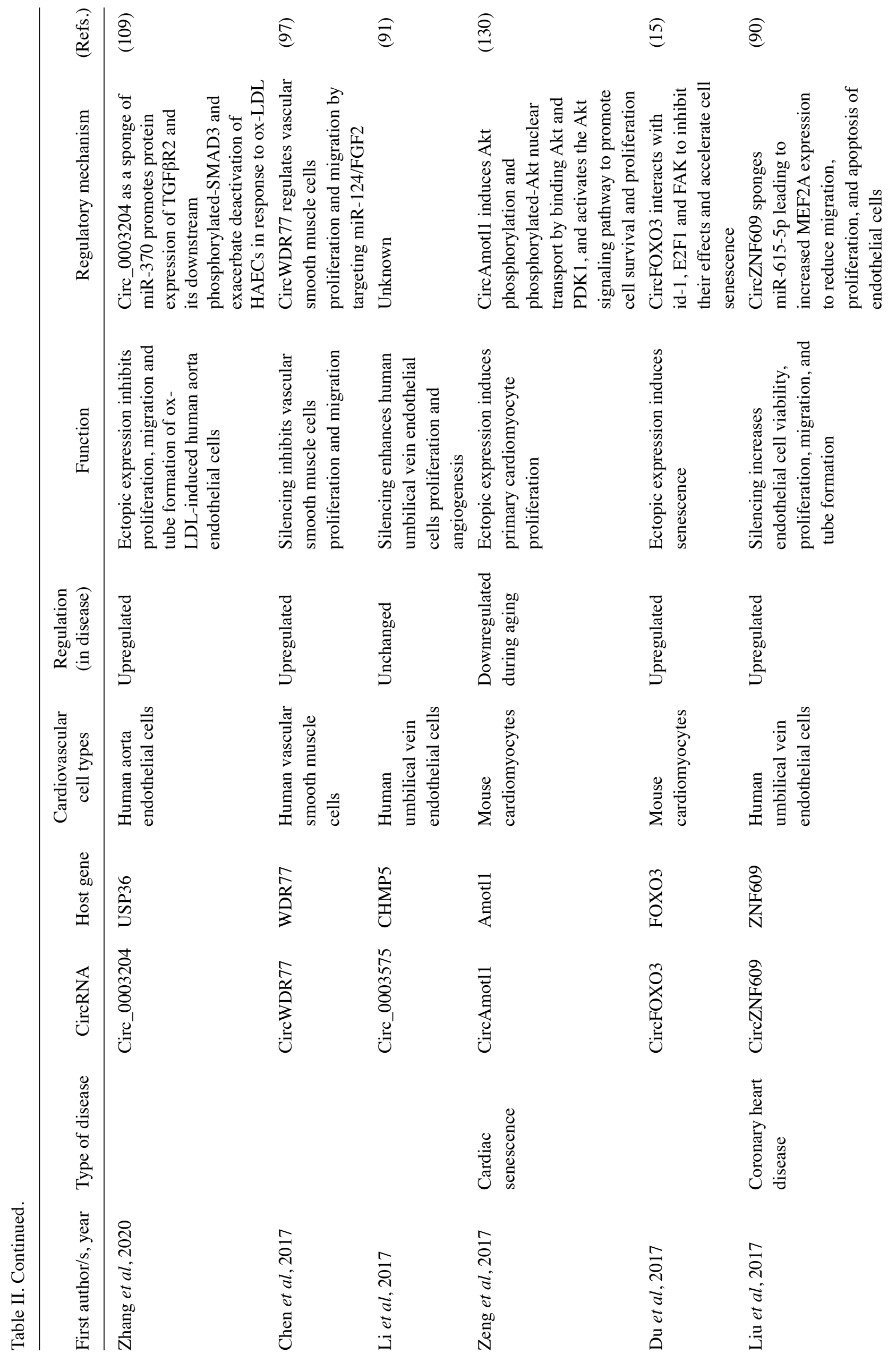




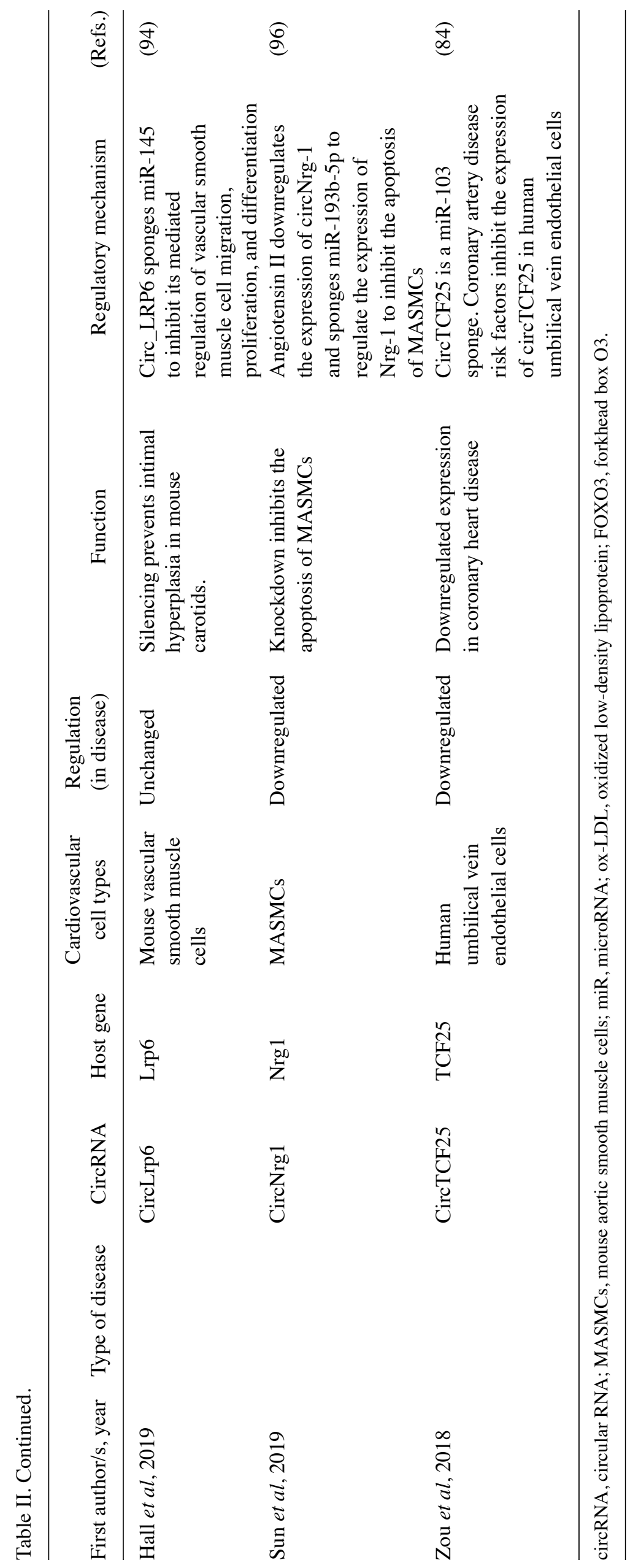




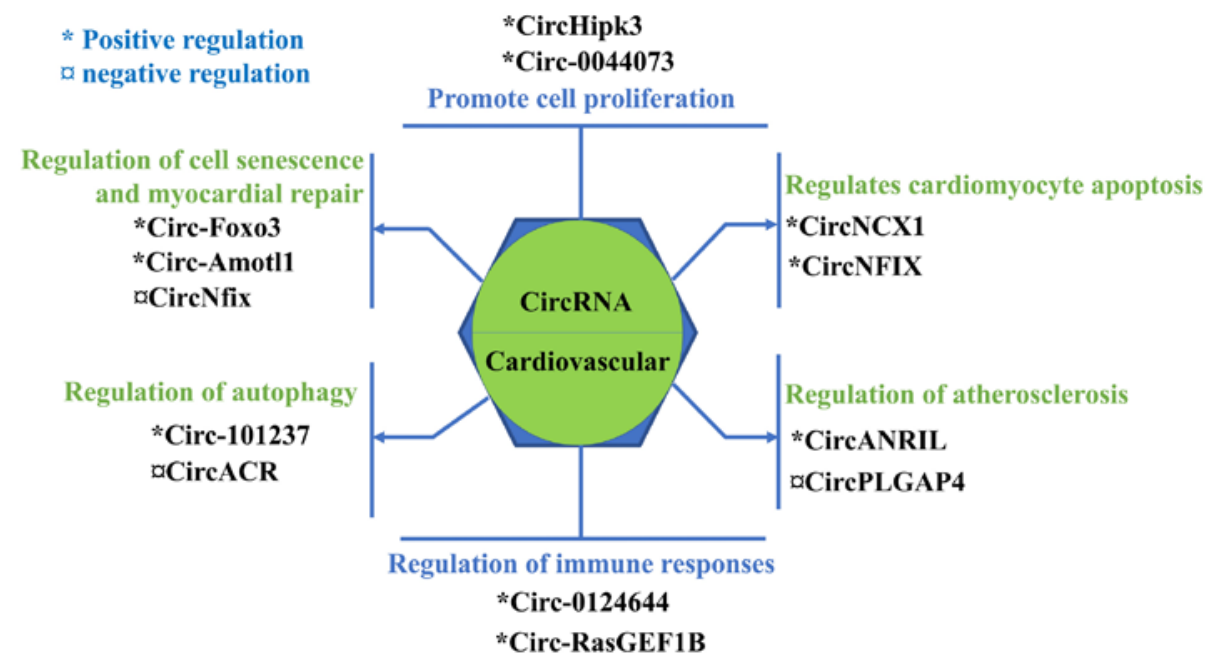

Figure 2. CircRNAs as therapeutic agents, targets and/or biomarkers in cardiovascular. The possible regulatory mechanisms linking circRNA to cardiovascular diseases. Circ/CircRNA, circular RNA; FOXO3, forkhead box O3; Nfix, nuclear factor 1 X-type; RasGEF1B, Ras-GEF domain-containing family member 1B.

respectively. Therefore, the role of circ-ANRIL in regulating AS should be further investigated. In addition, a study revealed that circ-DLG associated protein 4 (DLGAP4) is negatively associated with the risk of coronary heart disease (118). Therefore, it was demonstrated that circ-DLGAP4 was downregulated in ox-LDL-induced foam cells in a time-dependent manner. In addition, circ-DLGAP4 could sponge endogenous miR-143 to reduce the size of the atherosclerotic plaque and macrophage infiltration in mice $(119,120)$. This finding indicated that circ-DLGAP4 could be considered as a protective factor against monocyte-macrophage foaming, and as a potential inhibitory factor of AS plaque forming.

Role of circRNAs in the regulation of immune responses. CircRNAs are involved in innate immunity (121). A study showed that circ_0124644 could affect the progression of CAD through regulating the immune responses such as cell adhesion, therefore, it is considered as a biomarker for CAD (105). Another study investigated the expression of circRNAs in bone marrow-derived macrophages under two distinct polarization conditions, namely M1 macrophages stimulated with interferon- $\gamma$ and LPS, and M2 macrophages induced by interleukin-4 (122). The results revealed an interaction between circRNA-010231 and miR-141-5p, miR-145a-5p, miR-1964-5p, miR-19b-2-5p and miR-6950-5p. circRNA-010231 regulated the expression of the aforementioned miRNAs via participating in the differentiation and polarization of macrophages, thereby regulating the immune responses. The circRNA Ras-GEF domain-containing family member 1B (RasGEF1B) predominantly exists in the cytoplasm of murine macrophages (123). Ng et al (123) demonstrated that the expression of intercellular adhesion molecule-1 (ICAM-1) is downregulated following circ-RasGEF1B knockout in LPS-induced macrophages, indicating that ICAM-1 is a downstream regulator of circ-RasGEF1B-associated immune responses. In addition, circ-RasGEF1B mediates the immune responses via regulating the stability of mature ICAM-1 mRNAs. Additional studies have also reported the role of different circRNAs on the regulation of the immune responses $(121,124,125)$. Therefore, the effects of circRNAs on the immune function in CVDs are worth further investigation.
Role of circRNAs in the regulation of autophagy. CircRNAs are involved in autophagy by regulating the transcription and post-transcriptional modifications of autophagy-related genes (126). Cell autophagy is considered as a double-edged sword in health and disease, while it exerts protective effects on restoring homeostasis, it has also been reported that excessive autophagy mediates cardiomyocyte death (127). Zhou et al (128) demonstrated that circ-acrosin (ACR) attenuates autophagy and cell death in cardiomyocytes. In addition, ACR protects against ischemia-reperfusion injury and reduces myocardial infarct sizes in a myocardial ischemia/reperfusion injury model. The same study revealed that circ-ACR could induce the expression of putative kinase 1 (PINK-1) via directly binding to DNA methyltransferase 3B (Dnmt3b), thus blocking the Dnmt3b-mediated DNA methylation of the PINK-1 promoter. Furthermore, PINK-1 could phosphorylate its downstream target, Rho family-interacting cell polarization regulator 2 on serine 46 and inhibit cardiac autophagy and cell death. Another study on autophagy demonstrated that circRNA_101237 acts as a sponge of let-7a-5p to regulate insulin like growth factor $2 \mathrm{mRNA}$ binding protein 3 (IGF2BP3)-dependent autophagy (106). Therefore, circRNA_101237 downregulation decreases the expression of IGF2BP3, which in turn attenuates the apoptosis of primary cardiomyocytes and inhibits hypoxia/reoxygenation-induced autophagy. The aforementioned studies indicated that autophagy could serve an important role in the regulation of CVDs.

Role of circRNAs in the regulation of cell senescence and myocardial repair. The senescence and repair of tissues or cells is closely associated with their function (107,129-131). A study showed that the circRNA forkhead box O3 (FOXO3) is significantly upregulated in heart samples of elderly patients and mice and is associated with cell senescence (15). Therefore, circ-FOXO3 is mainly distributed in the cytoplasm, where it can interact with the anti-senescence protein inhibitor of DNA binding-1, the transcription factor E2F1 and the anti-stress proteins focal adhesion kinase and HIF-1 $\alpha$, thereby promoting cardiomyocyte senescence. Additional mechanistic studies suggested that the pro-senescent effect of circ-FOXO3 
could be associated with the regulation of the cell cycle. Therefore, in this study, the ectopically expressed circ-FOXO3 could form a ternary complex by binding with cyclin kinase 2 (CDK2) and cycle dependent kinase inhibitor 1 (p21). The formed circ-FOXO3-p21-CDK2 ternary complex blocks the function of CDK2, thereby inhibiting the progression of the cell cycle (129). Zeng et al (130) investigated the role of circRNA in myocardial repair by screening the myocardial tissues of patients of different ages. The results revealed that circRNA angiomotin Like 1 (Amotl1) is significantly upregulated in myocardial tissues, thus enhancing the myocardial function in the neonatal heart. Furthermore, circ-Amotl1 induces the phosphorylation of Akt and its nuclear transportation via binding Akt and phosphoinositide dependent kinase 1. This process promotes the activation of the Akt signaling pathway, resulting in enhanced cell survival and proliferation. In addition, circ-Amotl1 could play a protective role in the DOX-induced cardiomyopathy (130). In another study, the authors investigated the positive and negative regulatory effects of the signaling pathways Meis1/circ-nuclear factor 1 X-type (Nfix)/miR-214/Gsk $3 \beta / \beta$-catenin and circ-Nfix/Ybx1/cyclin A2 and cyclin B1 by functional verification experiments (107). The results revealed a close association between the expression of circ-Nfix and heart regeneration and repair. The aforementioned studies indicated that circRNAs could serve an important role in regulating cell senescence and cell regeneration, and could be used as targets for treating heart failure and myocardial infarction in the future.

\section{Perspectives and conclusions}

Nowadays, the interest of the scientific community regarding circRNAs is increasing with their comprehensive exploration, and the mystery of circRNAs has been slowly revealed. Although several functions of circRNAs have been identified, numerous remain to be elucidated. Due to their high stability and resistance to RNases, circRNAs may accumulate in quiescent or post-mitotic cells, such as neurons (132). Several studies revealed that the expression of circRNAs in proliferative cells and tissues, including cancer cells, is lower compared with terminally differentiated cells, indicating a negative association between circRNA abundance and cell proliferation (133). Previous studies have shown that circRNAs are enriched in extracellular vesicles and are secreted by cells in the form of vesicles (133-137). Notably, smaller circRNAs are more prone to vesicle-mediated transport (35). In addition, circRNAs maintain their circular structure in vesicles and perform their activities in recipient cells, suggesting that circRNA transport may be important for cell-to-cell communication (136). In cardiac metabolic diseases, serum markers in peripheral blood, such as cardiac troponin and creatine kinase, serve a significant role in the diagnosis of several diseases. It has been reported that certain linear non-coding RNAs can be used as biomarkers in the diagnosis of CAD $(137,138)$. Therefore, circRNAs could be considered as potential biomarkers for the diagnosis and treatment of CVDs. However, whether circRNAs selectively enter into exosomes, and the mechanism and significance of exosomal circRNA formation should be verified first. Certain circRNAs accumulate in the nucleus, while the majority are effectively transported to the cytoplasm (41). The accumulation of circRNAs in the nucleus is mediated by the ATP-dependent RNA helicase DexH/D-box, and their nuclear export depends on the length of the mature circRNA (139). m6A modification widely occurs on circRNAs (140). In view of the influence of m6A-binding protein YTH domain-containing 1 (YTHDC1) on the nuclear export of methylated mRNA (141), the effect of m6A on the export of circRNAs remains to be elucidated, to the best of our knowledge. Several circRNA-mediated degradation mechanisms have been reported $(121,142,143)$. Therefore, the binding of circRNAs by miRNAs can initiate circRNA decay (121). Furthermore, circRNA can also be degraded by the cytoplasmic endonuclease RNase L, which is activated in the presence of pathogenic double-strand RNA during viral infection (142). In addition, it has been reported that the YTHDF2-HRSP12-RNase P/MRP and UPF1-G3BP1 complexes mediate m6A-modified and high-structured circRNAs, respectively (143). However, to the best of our knowledge, how the degradation mechanisms of circRNAs affect the disease course remains to be investigated. It is understood that circRNAs are conserved among various species (75), and are specifically expressed in different tissues and different developmental stages $(12,76)$. Nevertheless, the reasons for the conservatism and specificity of circRNAs and how they affect the functional development of tissues and organs have not been reported to date. Although it has been documented that circRNAs exert a folded secondary structure (144), to the best of our knowledge, the association between their secondary structure and their function remains unclear, and it is worthy of further investigation. There are a number of limited reports regarding the translational function of circRNAs, including the IRES and m6A modification pathways $(52,53,81,145)$. Recently, researchers have combined the stability of circRNAs and their potential for continuous translation to develop a new expression system that could produce a protein in a sustainable and stable manner (146). This finding indicated that the translational function of circRNAs could be applied in the future for biological therapy.

A number of circRNAs have been identified, discovered and applied in the CAD $(84,105,118)$. It has been suggested that active pharmaceutical ingredients can interfere with the differential expression of circRNAs in CAD (147). Several networks of circRNA-miRNA signaling pathways in the CAD have been also reported $(103,148)$. These networks are systematic and three-dimensional and can truly and dynamically reflect the occurrence and development of CAD. A study revealed that circRNAs could be involved in chemoresistance, thus providing a novel approach for circRNAs in drug intervention and targeted therapy for CVDs (149). In addition, a research team used RNA polymers and poly (lactic-co-glycolic acid) (PLGA) coated stents containing and releasing therapeutic factors to treat local lesions in AS (149). The results demonstrated that these stents could promote endothelial cell regeneration and reduce the risk of coronary artery restenosis. Another study suggested that modern transfection methods could be used to transfer circRNAs during cardiovascular operations (150). Recently, a study introduced the circmiRs technology, opening a new chapter in the in vivo treatment of CAD using circRNAs (151). In this study, a circmiR sponge was constructed targeting the known cardiac pro-hypertrophic miRs-132 and miRs-212. Therefore, the hypertrophic 
characteristics of the disease were attenuated, and the cardiac function was preserved in transverse aortic constriction-treated mice, thus supporting the potential of circmiRs as novel therapeutic tools. By exploiting the characteristics of circRNAs in CVDs through drug intervention, artificial overexpression or knockout, and circRNA sequencing, it is possible to achieve a more accurate treatment strategy for CVDs.

\section{Acknowledgements}

Not applicable.

\section{Funding}

The present study was supported by the Key Scientific and Technological Project of Meizhou People's Hospital (grant no. MPHKSTP-20180101 to Dr ZZ), the Guangdong Provincial Key Laboratory of Precision Medicine and Clinical Translation Research of Hakka Population (grant no. 2018B030322003) and the Science and Technology Program of Meizhou (grant no. 2019B0202001).

\section{Availability of data and materials}

Not applicable.

\section{Authors' contributions}

ZY, HW and ZZ contributed to the conception of the study, performed the literature search and wrote the manuscript. $\mathrm{QH}$, QZ and HW edited the manuscript, assisted in the literature search and critically revised the article for important intellectual content. All the authors have read and approved the final manuscript.

\section{Ethics approval and consent to participate}

Not applicable.

\section{Patient consent for publication}

Not applicable.

\section{Competing interests}

The authors declare that they have no competing interests.

\section{References}

1. Pennisi E: Shining a light on the genome's 'dark matter'. Science 330: 1614, 2010.

2. Mattick JS and Makunin IV: Non-coding RNA. Hum Mol Genet 15 (Suppl 1): R17-R29, 2006.

3. Memczak S, Jens M, Elefsinioti A, Torti F, Krueger J, Rybak A, Maier L, Mackowiak SD, Gregersen LH, Munschauer M, et al: Circular RNAs are a large class of animal RNAs with regulatory potency. Nature 495: 333-338, 2013.

4. Hsu MT and Coca-Prados M: Electron microscopic evidence for the circular form of RNA in the cytoplasm of eukaryotic cells Nature 280: 339-340, 1979.

5. Sanger HL, Klotz G, Riesner D, Gross HJ and Kleinschmidt AK : Viroids are single-stranded covalently closed circular RNA molecules existing as highly base-paired rod-like structures. Proc Natl Acad Sci USA 73: 3852-3856, 1976.
6. Salzman J, Chen RE, Olsen MN, Wang PL and Brown PO: Cell-type specific features of circular RNA expression. PLoS Genet 9: e1003777, 2013.

7. Wang PL, Bao Y, Yee MC, Barrett SP, Hogan GJ, Olsen MN, Dinneny JR, Brown PO and Salzman J: Circular RNA is expressed across the eukaryotic tree of life. PLoS One 9: e90859, 2014.

8. Jeck WR and Sharpless NE: Detecting and characterizing circular RNAs. Nat Biotechnol 32: 453-461, 2014.

9. Hansen TB, Jensen TI, Clausen BH, Bramsen JB, Finsen B, Damgaard CK and Kjems J: Natural RNA circles function as efficient microRNA sponges. Nature 495: 384-388, 2013.

10. Dong R, Ma XK, Li GW and Yang L: CIRCpedia v2: An updated database for comprehensive circular RNA annotation and expression comparison. Genomics Proteomics Bioinformatics 16: 226-233, 2018.

11. Han B, Chao J and Yao H: Circular RNA and its mechanisms in disease: From the bench to the clinic. Pharmacol Ther 187: 31-44, 2018.

12. Siede D, Rapti K, Gorska AA, Katus HA, Altmüller J, Boeckel JN, Meder B, Maack C, Völkers M, Müller OJ, et al: Identification of circular RNAs with host gene-independent expression in human model systems for cardiac differentiation and disease. J Mol Cell Cardiol 109: 48-56, 2017

13. Zhang F, Zhang R, Zhang X, Wu Y, Li X, Zhang S, Hou W, Ding Y, Tian J, Sun L and Kong X: Comprehensive analysis of circRNA expression pattern and circRNA-miRNA-mRNA network in the pathogenesis of atherosclerosis in rabbits. Aging (Albany NY) 10: 2266-2283, 2018.

14. Santos-Ribeiro D, Godinas L, Pilette C and Perros F: The integrated stress response system in cardiovascular disease. Drug Discov Today 23: 920-929, 2018

15. Du WW, Yang W, Chen Y, Wu ZK, Foster FS, Yang Z, Li X and Yang BB: Foxo3 circular RNA promotes cardiac senescence by modulating multiple factors associated with stress and senescence responses. Eur Heart J 38: 1402-1412, 2017.

16. Pan T, Sun X, Liu Y, Li H, Deng G, Lin H and Wang S: Heat stress alters genome-wide profiles of circular RNAs in Arabidopsis. Plant Mol Biol 96: 217-229, 2018.

17. Ashwal-Fluss R, Meyer M, Pamudurti NR, Ivanov A, Bartok O, Hanan M, Evantal N, Memczak S, Rajewsky N and Kadener S: circRNA biogenesis competes with pre-mRNA splicing. Mol Cell 56: 55-66, 2014

18. Fu XD and Ares M Jr: Context-dependent control of alternative splicing by RNA-binding proteins. Nat Rev Genet 15: 689-701, 2014.

19. Starke S, Jost I, Rossbach O, Schneider T, Schreiner S, Hung LH and Bindereif A: Exon circularization requires canonical splice signals. Cell Rep 10: 103-111, 2015.

20. Kramer MC, Liang D, Tatomer DC, Gold B, March ZM, Cherry S and Wilusz JE: Combinatorial control of drosophila circular RNA expression by intronic repeats, hnRNPs, and SR proteins. Genes Dev 29: 2168-2182, 2015.

21. Conn SJ, Pillman KA, Toubia J, Conn VM, Salmanidis M, Phillips CA, Roslan S, Schreiber AW, Gregory PA and Goodall GJ: The RNA binding protein quaking regulates formation of circRNAs. Cell 160: 1125-1134, 2015

22. Errichelli L, Dini Modigliani S, Laneve P, Colantoni A, Legnini I, Capauto D, Rosa A, De Santis R, Scarfò R, Peruzzi G, et al: FUS affects circular RNA expression in murine embryonic stem cell-derived motor neurons. Nat Commun 8: 14741, 2017.

23. Jeck WR, Sorrentino JA, Wang K, Slevin MK, Burd CE, Liu J, Marzluff WF and Sharpless NE: Circular RNAs are abundant, conserved, and associated with ALU repeats. RNA 19: 141-157, 2013.

24. Ivanov A, Memczak S, Wyler E, Torti F, Porath HT, Orejuela MR, Piechotta M, Levanon EY, Landthaler M, Dieterich C and Rajewsky N: Analysis of intron sequences reveals hallmarks of circular RNA biogenesis in animals. Cell Rep 10: 170-177, 2015.

25. Lander ES, Linton LM, Birren B, Nusbaum C, Zody MC, Baldwin J, Devon K, Dewar K, Doyle M, FitzHugh W, et al: Initial sequencing and analysis of the human genome. Nature 409: 860-921, 2001.

26. Sela N, Mersch B, Gal-Mark N, Lev-Maor G, Hotz-Wagenblatt A and Ast G: Comparative analysis of transposed element insertion within human and mouse genomes reveals Alu's unique role in shaping the human transcriptome. Genome Biol 8: R127, 2007.

27. Liang D and Wilusz JE: Short intronic repeat sequences facilitate circular RNA production. Genes Dev 28: 2233-2247, 2014.

28. Ruskin B and Green MR: An RNA processing activity that debranches RNA lariats. Science 229: 135-140, 1985. 
29. Aufiero S, van den Hoogenhof MMG, Reckman YJ, Beqqali A van der Made I, Kluin J, Khan MAF, Pinto YM and Creemers EE: Cardiac circRNAs arise mainly from constitutive exons rather than alternatively spliced exons. RNA 24: 815-827, 2018.

30. Salzman J, Gawad C, Wang PL, Lacayo N and Brown PO: Circular RNAs are the predominant transcript isoform from hundreds of human genes in diverse cell types. PLoS One 7: e30733, 2012.

31. Talhouarne GJS and Gall JG: Lariat intronic RNAs in the cytoplasm of vertebrate cells. Proc Natl Acad Sci USA 115: E7970-E7977, 2018

32. Noto JJ, Schmidt CA and Matera AG: Engineering and expressing circular RNAs via tRNA splicing. RNA Biol 14: 978-984, 2017.

33. Pasman Z, Been MD and Garcia-Blanco MA: Exon circularization in mammalian nuclear extracts. RNA 2: 603-610, 1996.

34. Lyu D and Huang S: The emerging role and clinical implication of human exonic circular RNA. RNA Biol 14: 1000-1006, 2017.

35. Li Y, Zheng Q, Bao C, Li S, Guo W, Zhao J, Chen D, Gu J, He X and Huang S: Circular RNA is enriched and stable in exosomes: A promising biomarker for cancer diagnosis. Cell Res 25: 981-984, 2015 .

36. Zhao RT, Zhou J, Dong XL, Bi CW, Jiang RC, Dong JF, Tian Y, Yuan HJ and Zhang JN: Circular ribonucleic acid expression alteration in exosomes from the brain extracellular space after traumatic brain injury in mice. J Neurotrauma 35: 2056-2066, 2018.

37. $\mathrm{Hu} \mathrm{Q}$ and Zhou T: EIciRNA-mediated gene expression: Tunability and bimodality. FEBS Lett 592: 3460-3471, 2018.

38. Panda AC, De S, Grammatikakis I, Munk R, Yang X, Piao Y Dudekula DB, Abdelmohsen K and Gorospe M: High-purity circular RNA isolation method (RPAD) reveals vast collection of intronic circRNAs. Nucleic Acids Res 45: e116, 2017.

39. Piwecka M, Glažar P, Hernandez-Miranda LR, Memczak S, Wolf SA, Rybak-Wolf A, Filipchyk A, Klironomos F, Cerda Jara CA, Fenske P, et al: Loss of a mammalian circular RNA locus causes miRNA deregulation and affects brain function. Science 357: eaam8526, 2017

40. Ji P, Wu W, Chen S, Zheng Y, Zhou L, Zhang J, Cheng H, Yan J, Zhang S, Yang P and Zhao F: Expanded expression landscape and prioritization of circular RNAs in mammals. Cell Rep 26 : 3444-3460.e5, 2019

41. Kristensen LS, Andersen MS, Stagsted LVW, Ebbesen KK, Hansen TB and Kjems J: The biogenesis, biology and characterization of circular RNAs. Nat Rev Genet 20: 675-691, 2019.

42. Guo JU, Agarwal V, Guo H and Bartel DP: Expanded identification and characterization of mammalian circular RNAs. Genome Biol 15: 409, 2014

43. Hansen TB, Wiklund ED, Bramsen JB, Villadsen SB Statham AL, Clark SJ and Kjems J: miRNA-dependent gene silencing involving Ago2-mediated cleavage of a circular antisense RNA. EMBO J 30: 4414-4422, 2011.

44. Kleaveland B, Shi CY, Stefano J and Bartel DP: A network of noncoding regulatory RNAs acts in the mammalian brain. Cell 174: 350-362.e17, 2018.

45. Chen LL: The biogenesis and emerging roles of circular RNAs Nat Rev Mol Cell Biol 17: 205-211, 2016.

46. Liang D, Tatomer DC, Luo Z, Wu H, Yang L, Chen LL, Cherry S and Wilusz JE: The output of protein-coding genes shifts to circular RNAs when the pre-mRNA processing machinery is limiting. Mol Cell 68: 940-954 e3, 2017.

47. Li Z, Huang C, Bao C, Chen L, Lin M, Wang X, Zhong G, Yu B, $\mathrm{Hu} \mathrm{W}$, Dai L, et al: Exon-intron circular RNAs regulate transcription in the nucleus. Nat Struct Mol Biol 22: 256-264, 2015.

48. Zhang Y, Zhang XO, Chen T, Xiang JF, Yin QF, Xing YH, Zhu S Yang L and Chen LL: Circular intronic long noncoding RNAs. Mol Cell 51: 792-806, 2013.

49. Gupta SK, Garg A, Bär C, Chatterjee S, Foinquinos A, Milting H, Streckfuß-Bömeke K, Fiedler J and Thum T: Quaking inhibits doxorubicin-mediated cardiotoxicity through regulation of cardiac circular RNA expression. Circ Res 122: 246-254, 2018.

50. Rossi F, Legnini I, Megiorni F, Colantoni A, Santini T, Morlando M, Di Timoteo G, Dattilo D, Dominici C and Bozzoni I: Circ-ZNF609 regulates G1-S progression in rhabdomyosarcoma. Oncogene 38: 3843-3854, 2019.

51. Cardona-Monzonís A, García-Giménez JL, Mena-Mollá S, Pareja-Galeano H, de la Guía-Galipienso F, Lippi G, Pallardó FV and Sanchis-Gomar F: Non-coding RNAs and coronary artery disease. Adv Exp Med Biol 1229: 273-285, 2020.

52. Legnini I, Di Timoteo G, Rossi F, Morlando M, Briganti F Sthandier O, Fatica A, Santini T, Andronache A, Wade M, et al: Circ-ZNF609 is a circular RNA that can be translated and functions in myogenesis. Mol Cell 66: 22-37.e9, 2017.
53. Yang Y, Fan X, Mao M, Song X, Wu P, Zhang Y, Jin Y, Yang Y, Chen LL, Wang Y, et al: Extensive translation of circular RNAs driven by $\mathrm{N}^{6}$-methyladenosine. Cell Res 27: 626-641, 2017.

54. Glažar P, Papavasileiou P and Rajewsky N: CircBase: A database for circular RNAs. RNA 20: 1666-1670, 2014

55. Panda AC, Dudekula DB, Abdelmohsen K and Gorospe M: Analysis of circular RNAs using the web tool CircInteractome. Methods Mol Biol 1724: 43-56, 2018.

56. Liu YC, Li JR, Sun CH, Andrews E, Chao RF, Lin FM, Weng SL, Hsu SD, Huang CC, Cheng C, et al: CircNet: A database of circular RNAs derived from transcriptome sequencing data. Nucleic Acids Res 44 (D1): D209-D215, 2016.

57. Zheng LL, Li JH, Wu J, Sun WJ, Liu S, Wang ZL, Zhou H, Yang JH and Qu LH: DeepBase v2.0: Identification, expression, evolution and function of small RNAs, LncRNAs and circular RNAs from deep-sequencing data. Nucleic Acids Res 44 (D1): D196-D202, 2016

58. Liu M, Wang Q, Shen J, Yang BB and Ding X: Circbank: A comprehensive database for circRNA with standard nomenclature. RNA Biol 16: 899-905, 2019.

59. Xia S, Feng J, Chen K, Ma Y, Gong J, Cai F, Jin Y, Gao Y, Xia L, Chang H, et al: CSCD: A database for cancer-specific circular RNAs. Nucleic Acids Res 46 (D1): D925-D929, 2018.

60. Ghosal S, Das S, Sen R, Basak P and Chakrabarti J: Circ2Traits: A comprehensive database for circular RNA potentially associated with disease and traits. Front Genet 4: 283, 2013.

61. Li JH, Liu S, Zhou H, Qu LH and Yang JH: starBase v2.0: Decoding miRNA-ceRNA, miRNA-ncRNA and protein-RNA interaction networks from large-scale CLIP-Seq data. Nucleic Acids Res 42 (Database Issue): D92-D97, 2014.

62. Wang D: hppRNA-a snakemake-based handy parameter-free pipeline for RNA-Seq analysis of numerous samples. Brief Bioinform 19: 622-626, 2018

63. Wu W, Ji P and Zhao F: CircAtlas: An integrated resource of one million highly accurate circular RNAs from 1070 vertebrate transcriptomes. Genome Biol 21: 101, 2020.

64. Cai Z, Fan Y, Zhang Z, Lu C, Zhu Z, Jiang T, Shan T and Peng Y: VirusCircBase: A database of virus circular RNAs. Brief Bioinform: Apr 29, 2020 (Epub ahead of print)

65. Chen X, Han P, Zhou T, Guo X, Song X and Li Y: circRNADb: A comprehensive database for human circular RNAs with protein-coding annotations. Sci Rep 6: 34985, 2016.

66. Chen X, Chen RX, Wei WS, Li YH, Feng ZH, Tan L, Chen JW, Yuan GJ, Chen SL, Guo SJ, et al: PRMT5 circular RNA promotes metastasis of urothelial carcinoma of the bladder through sponging miR-30c to induce epithelial-mesenchymal transition. Clin Cancer Res 24: 6319-6330, 2018.

67. Li T, Shao Y, Fu L, Xie Y, Zhu L, Sun W, Yu R, Xiao B and Guo J: Plasma circular RNA profiling of patients with gastric cancer and their droplet digital RT-PCR detection. J Mol Med (Berl) 96: 85-96, 2018.

68. Chen DF, Zhang LJ, Tan K and Jing Q: Application of droplet digital PCR in quantitative detection of the cell-free circulating circRNAs. Biotechnol Biotechnol Equip 32: 116-123, 2018.

69. Wang K, Long B, Liu F, Wang JX, Liu CY, Zhao B, Zhou LY, Sun T, Wang M, Yu T, et al: A circular RNA protects the heart from pathological hypertrophy and heart failure by targeting miR-223. Eur Heart J 37: 2602-2611, 2016.

70. Kamens J: The addgene repository: An international nonprofit plasmid and data resource. Nucleic Acids Res 43 (Database Issue): D1152-D1157, 2015.

71. Boeckel JN, Jaé N, Heumüller AW, Chen W, Boon RA, Stellos K, Zeiher AM, John D, Uchida S and Dimmeler S: Identification and characterization of hypoxia-regulated endothelial circular RNA. Circ Res 117: 884-890, 2015.

72. Bramsen JB, Laursen MB, Nielsen AF, Hansen TB, Bus C, Langkjaer N, Babu BR, Højland T, Abramov M, Van Aerschot A, et al: A large-scale chemical modification screen identifies design rules to generate siRNAs with high activity, high stability and low toxicity. Nucleic Acids Res 37: 2867-2881, 2009

73. de Bruyns A, Geiling B and Dankort D: Construction of modular lentiviral vectors for effective gene expression and knockdown. Methods Mol Biol 1448: 3-21, 2016.

74. Jakobi T, Czaja-Hasse LF, Reinhardt R and Dieterich C: Profiling and validation of the circular RNA repertoire in adult murine hearts. Genomics Proteomics Bioinformatics 14: 216-223, 2016.

75. Werfel S, Nothjunge S, Schwarzmayr T, Strom TM, Meitinger T and Engelhardt S: Characterization of circular RNAs in human, mouse and rat hearts. J Mol Cell Cardiol 98: 103-107, 2016 
76. Tan WL, Lim BT, Anene-Nzelu CG, Ackers-Johnson M, Dashi A See K, Tiang Z, Lee DP, Chua WW, Luu TD, et al: A landscape of circular RNA expression in the human heart. Cardiovasc Res 113: 298-309, 2017.

77. Lei W, Feng T, Fang X, Yu Y, Yang J, Zhao ZA, Liu J, Shen Z, Deng W and Hu S: Signature of circular RNAs in human induced pluripotent stem cells and derived cardiomyocytes. Stem Cell Res Ther 9: 56, 2018.

78. Li Y, Zhang J, Huo C, Ding N, Li J, Xiao J, Lin X, Cai B, Zhang Y and Xu J: Dynamic organization of lncRNA and circular RNA regulators collectively controlled cardiac differentiation in humans. EBioMedicine 24: 137-146, 2017.

79. Stagsted LV, Nielsen KM, Daugaard I and Hansen TB: Noncoding AUG circRNAs constitute an abundant and conserved subclass of circles. Life Sci Alliance 2: e201900398, 2019.

80. Jakobi T, Siede D, Eschenbach J, Heumüller AW, Busch M, Nietsch R, Meder B, Most P, Dimmeler S, Backs J, et al: Deep characterization of circular RNAs from human cardiovascular cell models and cardiac tissue. Cells 9: 1616, 2020

81. van Heesch S, Witte F, Schneider-Lunitz V, Schulz JF, Adami E, Faber AB, Kirchner M, Maatz H, Blachut S, Sandmann CL, et al: The translational landscape of the human heart. Cell 178: 242-260.e29, 2019

82. Wang K, Gan TY, Li N, Liu CY, Zhou LY, Gao JN, Chen C, Yan KW, Ponnusamy M, Zhang YH and Li PF: Circular RNA mediates cardiomyocyte death via miRNA-dependent upregulation of MTP18 expression. Cell Death Differ 24: 1111-1120, 2017.

83. Wang L, Shen C, Wang Y, Zou T, Zhu H, Lu X, Li L, Yang B, Chen J, Chen S, et al: Identification of circular RNA Hsa circ_0001879 and Hsa_circ_0004104 as novel biomarkers for coronary artery disease. Atherosclerosis 286: 88-96, 2019.

84. Zou TY, Li L, Huang JF, Yang B and Wang LY: Expression and clinical significance of circular RNA circTCF25 in patients with coronary artery disease. Mol Cardiol China 3: 27-31, 2018

85. Holdt LM, Stahringer A, Sass K, Pichler G, Kulak NA, Wilfert W, Kohlmaier A, Herbst A, Northoff BH, Nicolaou A, et al: Circular non-coding RNA ANRIL modulates ribosomal RNA maturation and atherosclerosis in humans. Nat Commun 7: 12429, 2016.

86. Hu X, Chen L, Wu S, Xu K, Jiang W, Qin M, Zhang Y and Liu X: Integrative analysis reveals key circular RNA in atrial fibrillation. Front Genet 10: 108, 2019.

87. Ludmer PL, Selwyn AP, Shook TL, Wayne RR, Mudge GH, Alexander RW and Ganz P: Paradoxical vasoconstriction induced by acetylcholine in atherosclerotic coronary arteries. $\mathrm{N}$ Engl J Med 315: 1046-1051, 1986.

88. Simionescu M: Implications of early structural-functional changes in the endothelium for vascular disease. Arterioscler Thromb Vasc Biol 27: 266-274, 2007.

89. Chen PY, Qin L, Baeyens N, Li G, Afolabi T, Budatha M, Tellides G, Schwartz MA and Simons $M$ : Endothelial-to-mesenchymal transition drives atherosclerosis progression. J Clin Invest 125: 4514-4528, 2015.

90. Liu C, Yao MD, Li CP, Shan K, Yang H, Wang JJ, Liu B, Li XM, Yao J, Jiang Q and Yan B: Silencing Of Circular RNA-ZNF609 ameliorates vascular endothelial dysfunction. Theranostics 7: 2863-2877, 2017

91. Li CY, Ma L and Yu B: Circular RNA hsa_circ_0003575 regulates oxLDL induced vascular endothelial cells proliferation and angiogenesis. Biomed Pharmacother 95: 1514-1519, 2017.

92. Yang L, Han B, Zhang Y, Bai Y, Chao J, Hu G and Yao H: Engagement of circular RNA HECW2 in the nonautophagic role of ATG5 implicated in the endothelial-mesenchymal transition. Autophagy 14: 404-418, 2018.

93. Liu C, Ge HM, Liu BH, Dong R, Shan K, Chen X, Yao MD, Li XM, Yao J, Zhou RM, et al: Targeting pericyte-endothelial cell crosstalk by circular RNA-cPWWP2A inhibition aggravates diabetes-induced microvascular dysfunction. Proc Natl Acad Sci USA 116: 7455-7464, 2019.

94. Hall IF, Climent M, Quintavalle M, Farina FM, Schorn T, Zani S, Carullo P, Kunderfranco P, Civilini E, Condorelli G and Elia L: Circ_Lrp6, a Circular RNA enriched in vascular smooth muscle cells, acts as a sponge regulating miRNA-145 function. Circ Res 124: 498-510,2019.

95. Zheng C, Niu H, Li M, Zhang H, Yang Z, Tian L, Wu Z, Li D and Chen X: Cyclic RNA has-circ-000595 regulates apoptosis of aortic smooth muscle cells. Mol Med Rep 12: 6656-6662, 2015.

96. Sun Y, Zhang S, Yue M, Li Y, Bi J and Liu H: Angiotensin II inhibits apoptosis of mouse aortic smooth muscle cells through regulating the circNRG-1/miR-193b-5p/NRG-1 axis. Cell Death Dis 10: 362,2019
97. Chen J, Cui L, Yuan J, Zhang Y and Sang H: Circular RNA WDR77 target FGF-2 to regulate vascular smooth muscle cells proliferation and migration by sponging miR-124. Biochem Biophys Res Commun 494: 126-132, 2017.

98. Sun Y, Yang Z,Zheng B,Zhang XH,Zhang ML,ZhaoXS,ZhaoHY, Suzuki T and Wen JK: A novel regulatory mechanism of smooth muscle $\alpha$-actin expression by NRG-1/circACTA2/miR-548f-5p Axis. Circ Res 121: 628-635, 2017.

99. Zhu Y, Pan W, Yang T, Meng X, Jiang Z, Tao L and Wang L: Upregulation of circular RNA circNFIB attenuates cardiac fibrosis by sponging miR-433. Front Genet 10: 564, 2019.

100. Sun LY, Zhao JC, Ge XM, Zhang H, Wang CM and Bie ZD: Circ LAS1L regulates cardiac fibroblast activation, growth, and migration through miR-125b/SFRP5 pathway. Cell Biochem Funct 38: 443-450, 2020 .

101. Zhou B and Yu JW: A novel identified circular RNA, circRNA_010567, promotes myocardial fibrosis via suppressing miR-141 by targeting TGF- $\beta 1$. Biochem Biophys Res Commun 487: 769-775, 2017.

102. Tang CM, Zhang M, Huang L, Hu ZQ, Zhu JN, Xiao Z, Zhang Z, Lin QX, Zheng XL, -Yang M, et al: CircRNA_000203 enhances the expression of fibrosis-associated genes by derepressing targets of miR-26b-5p, Colla 2 and CTGF, in cardiac fibroblasts. Sci Rep 7: 40342, 2017

103. Shen L, Hu Y, Lou J, Yin S, Wang W, Wang Y, Xia Y and Wu W: CircRNA-0044073 is upregulated in atherosclerosis and increases the proliferation and invasion of cells by targeting miR-107. Mol Med Rep 19: 3923-3932, 2019.

104. Geng HH, Li R, Su YM, Xiao J, Pan M, Cai XX and Ji XP: The circular RNA Cdrlas promotes myocardial infarction by mediating the regulation of miR-7a on its target genes expression. PLoS One 11: e0151753, 2016.

105. Zhao Z, Li X, Gao C, Jian D, Hao P, Rao L and Li M: Peripheral blood circular RNA hsa_circ_0124644 can be used as a diagnostic biomarker of coronary artery disease. Sci Rep 7: 39918, 2017.

106. Gan J, Yuan J, Liu Y, Lu Z, Xue Y, Shi L and Zeng H: Circular RNA_101237 mediates anoxia/reoxygenation injury by targeting let-7a-5p/IGF2BP3 in cardiomyocytes. Int J Mol Med 45: 451-460, 2020

107. Huang S, Li X, Zheng H, Si X, Li B, Wei G, Li C, Chen Y, Chen Y, Liao W, et al: Loss of super-enhancer-regulated circRNA Nfix induces cardiac regeneration after myocardial infarction in adult mice. Circulation 139: 2857-2876, 2019.

108. Lim TB, Aliwarga E, Luu TDA, Li YP, Ng SL, Annadoray L, Sian S, Ackers-Johnson MA and Foo RS: Targeting the highly abundant circular RNA circSlc8a1 in cardiomyocytes attenuates pressure overload induced hypertrophy. Cardiovasc Res 115: 1998-2007, 2019.

109. Zhang S, Song G, Yuan J, Qiao S, Xu S, Si Z, Yang Y, Xu X and Wang A: Circular RNA circ_0003204 inhibits proliferation, migration and tube formation of endothelial cell in atherosclerosis via miR-370-3p/TGF $3 R 2 /$ phosph-SMAD3 axis. J Biomed Sci 27: 11,2020

110. Altesha MA, Ni T, Khan A, Liu K and Zheng X: Circular RNA in cardiovascular disease. J Cell Physiol 234: 5588-5600, 2019.

111. Ni H, Li W, Zhuge Y, Xu S, Wang Y, Chen Y, Shen G and Wang F: Inhibition of circHIPK3 prevents angiotensin II-induced cardiac fibrosis by sponging miR-29b-3p. Int J Cardiol 292: 188-196, 2019

112. Wang Y,Zhao R, Liu W, Wang Z, Rong J, Long X, Liu Z, Ge J and Shi B: Exosomal circHIPK3 released from hypoxia-pretreated cardiomyocytes regulates oxidative damage in cardiac microvascular endothelial cells via the miR-29a/IGF-1 pathway. Oxid Med Cell Longev 2019: 7954657, 2019.

113. Li M, Ding W, Tariq MA, Chang W, Zhang X, Xu W, Hou L, Wang Y and Wang J: A circular transcript of ncx1 gene mediates ischemic myocardial injury by targeting miR-133a-3p. Theranostics 8: 5855-5869, 2018.

114. Jin Q and Chen Y: Silencing circular RNA circ_0010729 protects human cardiomyocytes from oxygen-glucose deprivation-induced injury by up-regulating microRNA-145-5p. Mol Cell Biochem 462: 185-194, 2019.

115. Cai L, Qi B, Wu X, Peng S, Zhou G, Wei Y, Xu J, Chen S and Liu S: Circular RNA Ttc3 regulates cardiac function after myocardial infarction by sponging miR-15b. J Mol Cell Cardiol 130: 10-22, 2019

116. Holdt LM, Beutner F, Scholz M, Gielen S, Gäbel G, Bergert H, Schuler G, Thiery J and Teupser D: ANRIL expression is associated with atherosclerosis risk at chromosome 9p21. Arterioscler Thromb Vasc Biol 30: 620-627, 2010 
117. Song CL, Wang JP, Xue X, Liu N, Zhang XH, Zhao Z, Liu JG, Zhang CP, Piao ZH, Liu Y and Yang YB: Effect of Circular ANRIL on the inflammatory response of vascular endothelial cells in a rat model of coronary atherosclerosis. Cell Physiol Biochem 42: 1202-1212, 2017.

118. Menglan LI, Siying HE, Jialing R, Bin L, Xiaokang Z and Fang Z: The possible protective role of circDLGAP4 from peripheral blood in coronary heart disease. Chin J Clin Lab Sci 37: 109-112, 2019.

119. Parahuleva MS, Euler G, Mardini A, Parviz B, Schieffer B, Schulz R and Aslam M: Identification of microRNAs as potential cellular monocytic biomarkers in the early phase of myocardial infarction: A pilot study. Sci Rep 7: 15974, 2017.

120. Sala F, Aranda JF, Rotllan N, Ramírez CM, Aryal B, Elia L, Condorelli G, Catapano AL, Fernández-Hernando $\mathrm{C}$ and Norata GD: MiR-143/145 deficiency attenuates the progression of atherosclerosis in Ldlr-/-mice. Thromb Haemost 112: 796-802, 2014.

121. Liu CX, Li X, Nan F, Jiang S, Gao X, Guo SK, Xue W, Cui Y, Dong K, Ding H, et al: Structure and degradation of circular RNAs regulate PKR activation in innate immunity. Cell 177 865-880.e21, 2019.

122. Zhang Y,Zhang Y,Li X, Zhang M and Lv K: Microarray analysis of circular RNA expression patterns in polarized macrophages. Int J Mol Med 39: 373-379, 2017.

123. Ng WL, Marinov GK, Liau ES, Lam YL, Lim YY and Ea CK: Inducible RasGEF1B circular RNA is a positive regulator of ICAM-1 in the TLR4/LPS pathway. RNA Biol 13: 861-871, 2016.

124. Chen YG, Kim MV, Chen X, Batista PJ, Aoyama S, Wilusz JE, Iwasaki A and Chang HY: Sensing self and foreign circular RNAs by intron identity. Mol Cell 67: 228-238.e5, 2017.

125. Li X, Liu CX, Xue W, Zhang Y, Jiang S, Yin QF, Wei J, Yao RW, Yang L and Chen LL: Coordinated circRNA biogenesis and function with NF90/NF110 in viral infection. Mol Cell 67: 214-227.e7, 2017.

126. Zhang J, Wang P, Wan L, Xu S and Pang D: The emergence of noncoding RNAs as heracles in autophagy. Autophagy 13: 1004-1024, 2017.

127. Valentim L, Laurence KM, Townsend PA, Carroll CJ, Soond S, Scarabelli TM, Knight RA, Latchman DS and Stephanou A Urocortin inhibits Beclin1-mediated autophagic cell death in cardiac myocytes exposed to ischaemia/reperfusion injury. J Mol Cell Cardiol 40: 846-852, 2006.

128. Zhou LY, Zhai M, Huang Y, Xu S, An T, Wang YH, Zhang RC, Liu CY, Dong YH, Wang M, et al: The circular RNA ACR attenuates myocardial ischemia/reperfusion injury by suppressing autophagy via modulation of the Pink1/ FAM65B pathway. Cell Death Differ 26: 1299-1315, 2019.

129. Du WW, Yang W, Liu E, Yang Z, Dhaliwal P and Yang BB Foxo3 circular RNA retards cell cycle progression via forming ternary complexes with p21 and CDK2. Nucleic Acids Res 44: 2846-2858, 2016.

130. Zeng Y, Du WW, Wu Y, Yang Z, Awan FM, Li X, Yang W, Zhang C, Yang Q, Yee A, et al: A circular RNA binds to and activates AKT phosphorylation and nuclear localization reducing apoptosis and enhancing cardiac repair. Theranostics 7 : 3842-3855, 2017.

131. Garikipati VNS, Verma SK, Cheng Z, Liang D, Truongcao MM, Cimini M, Yue Y, Huang G, Wang C, Benedict C, et al: Circular RNA CircFndc $3 b$ modulates cardiac repair after myocardial infarction via FUS/VEGF-A axis. Nat Commun 10: 4317, 2019.

132. Westholm JO, Miura P, Olson S, Shenker S, Joseph B, Sanfilippo P, Celniker SE, Graveley BR and Lai EC: Genome-wide analysis of drosophila circular RNAs reveals their structural and sequence properties and age-dependent neural accumulation. Cell Rep 9: 1966-1980, 2014.

133. Bachmayr-Heyda A, Reiner AT, Auer K, Sukhbaatar N, Aust S, Bachleitner-Hofmann T, Mesteri I, Grunt TW, Zeillinger R and Pils D: Correlation of circular RNA abundance with proliferation-exemplified with colorectal and ovarian cancer, idiopathic lung fibrosis, and normal human tissues. Sci Rep 5: 8057, 2015.
134. Lasda E and Parker R: Circular RNAs co-precipitate with extracellular vesicles: A possible mechanism for circRNA clearance. PLoS One 11: e0148407, 2016.

135. Dou Y, Cha DJ, Franklin JL, Higginbotham JN, Jeppesen DK, Weaver AM, Prasad N, Levy S, Coffey RJ, Patton JG and Zhang B: Circular RNAs are down-regulated in KRAS mutant colon cancer cells and can be transferred to exosomes. Sci Rep 6: 37982, 2016.

136. Preußer C, Hung LH, Schneider T, Schreiner S, Hardt M, Moebus A, Santoso S and Bindereif A: Selective release of circRNAs in platelet-derived extracellular vesicles. J Extracell Vesicles 7: 1424473, 2018.

137. Viereck $\mathrm{J}$ and Thum T: Circulating noncoding RNAs as biomarkers of cardiovascular disease and injury. Circ Res 120 381-399, 2017.

138. Memczak S, Papavasileiou P, Peters O and Rajewsky N: Identification and characterization of circular RNAs as a new class of putative biomarkers in human blood. PLoS One 10 e0141214, 2015.

139. Huang C, Liang D, Tatomer DC and Wilusz JE: A length-dependent evolutionarily conserved pathway controls nuclear export of circular RNAs. Genes Dev 32: 639-644, 2018.

140. Zhou C, Molinie B, Daneshvar K, Pondick JV, Wang J, Van Wittenberghe N, Xing Y, Giallourakis CC and Mullen AC: Genome-wide maps of m6A circRNAs identify widespread and cell-type-specific methylation patterns that are distinct from mRNAs. Cell Rep 20: 2262-2276, 2017.

141. Roundtree IA, Luo GZ, Zhang Z, Wang X, Zhou T, Cui Y, Sha J, Huang X, Guerrero L, Xie P, et al: YTHDC1 mediates nuclear export of $\mathrm{N}^{6}$-methyladenosine methylated mRNAs. Elife 6 : e31311, 2017.

142. Han Y, Donovan J, Rath S, Whitney G, Chitrakar A and Korennykh A: Structure of human RNase L reveals the basis for regulated RNA decay in the IFN response. Science 343 1244-1248, 2014.

143. Park OH, Ha H, Lee Y, Boo SH, Kwon DH, Song HK and Kim YK: Endoribonucleolytic cleavage of $\mathrm{m}^{6} \mathrm{~A}$-containing RNAs by RNase P/MRP complex. Mol Cell 74: 494-507.e8, 2019

144. Tapsin S, Sun M, Shen Y, Zhang H, Lim XN, Susanto TT, Yang SL, Zeng GS, Lee J, Lezhava A, et al: Genome-wide identification of natural RNA aptamers in prokaryotes and eukaryotes. Nat Commun 9: 1289, 2018.

145. Yang Y, Gao X, Zhang M, Yan S, Sun C, Xiao F, Huang N, Yang X, Zhao K, Zhou H, et al: Novel role of FBXW7 Circular RNA in repressing glioma tumorigenesis. J Natl Cancer Inst 110: 304-315, 2018

146. Costello A, Lao NT, Barron N and Clynes M: Continuous translation of circularized mRNA improves recombinant protein titer. Metab Eng 52: 284-292, 2019.

147. Xie YZ, Yang F, Tan W, Li X, Jiao C, Huang R and Yang BB The anti-cancer components of Ganoderma lucidum possesses cardiovascular protective effect by regulating circular RNA expression. Oncoscience 3: 203-207, 2016

148. Dang RY, Liu FL and Li Y: Circular RNA hsa circ 0010729 regulates vascular endothelial cell proliferation and apoptosis by targeting the miR-186/HIF-1 $\alpha$ axis. Biochem Biophys Res Commun 490: 104-110, 2017.

149. Wei L, Sun J, Zhang N, Zheng Y, Wang X, Lv L, Liu J, Xu Y, Shen $Y$ and Yang $M$ : Noncoding RNAs in gastric cancer: Implications for drug resistance. Mol Cancer 19: 62, 2020.

150.Zhang Y, Yu J, Kahkoska AR and Gu Z: Photoacoustic drug delivery. Sensors (Basel) 17: 1400, 2017.

151. Lavenniah A,LuuTDA,Li YP,Lim TB,Jiang J,Ackers-Johnson M and Foo RS: Engineered circular RNA sponges act as miRNA inhibitors to attenuate pressure overload-induced cardiac hypertrophy. Mol Ther 28: 1506-1517, 2020.

This work is licensed under a Creative Commons Attribution-NonCommercial-NoDerivatives 4.0 International (CC BY-NC-ND 4.0) License. 